\title{
Identificação de pacientes com hipertensão resistente e pseudorresistente em uma Unidade de Saúde da Família
}

\author{
Identification of patients with resistant and pseudo-resistant \\ hypertension at a Family Health Unit
}

Recebido em: 16/01/2017

Aceito em: $\quad 06 / 06 / 2017$
Felipe Vieira da SILVA; Martha Gabriela Zambrana de AMORIM;

Alfredo Dias de OLIVEIRA FILHO; Sabrina Joany Felizardo NEVES

Núcleo de Estudos em Farmacoterapia - NEF. Escola de Enfermagem e Farmácia,

Universidade Federal de Alagoas - Campus A.C Simões. Avenida Lourival Melo Mota, Cidade Universitária, CEP 57072-900.Maceió, AL, Brasil.E-mail:fe_vs1@hotmail.com

\section{ABSTRACT}

Resistant Hypertension manifests when blood pressure (BP) remains above the recommended levels, despite regular use of three classes of antihypertensive drugs, including diuretics. This study aimed to identify resistant hypertension and pseudo-resistant hypertension in patients enrolled in a Family Health Unit. It was a cross-sectional study in hypertensive patients using antihypertensive medications seen at Basic Health Unit (BHU), located in the city of Maceio, through interviews with a structured questionnaire during home visits, as well as searching information from patients medical records at BHU. Patients were monitored for three consecutive weeks. The adherence was determined by applying the Morisky Adherence Scale Therapeutics eight items (MMAS-8). The antihypertensive drug therapy was evaluated according to the Brazilian Guidelines on Hypertension. Fifty 50 patients were investigated, with mean age of 66.4 years $(\mathrm{SD}=13.5)$. Most hypertensive patients studied were female $(74 \%)$. Among the patients, 39 were classified as pseudo-resistant hypertension (78\%), and 28 patients used antihypertensive regimens are considered inappropriate and 11 were non-adherent to treatment. In this study, $8 \%$ of hypertensive patients were diagnosed with resistant hypertension. Regarding SBP and DBP, gender, smoking habits and physical activity were not influencing factors. On the other hand, the proper prescription was correlated with the control of SBP, but not with the DBP control. The degree of adherence influenced both SBP and DBP. It was also observed the influence of subtype of hypertension on the SBP and DBP.

Key Words: medication adherence; hypertension; inappropriate prescribing

\section{RESUMO}

A Hipertensão Resistente (HAR) se manifesta quando os valores de pressão arterial (PA) permanecem acima da meta, apesar do uso regular de três classes de fármacos anti-hipertensivos, incluindo um diurético. Este estudo teve como objetivo identificar a HAR e a hipertensão pseudo-resistente em pacientes atendidos em uma Unidade de Saúde da Família. Foi realizado um estudo transversal com pacientes hipertensos em uso de medicamentos anti-hipertensivos atendidos em uma Unidade Básica de Saúde localizada no município de Maceió, por meio de entrevistas, com aplicação de questionário estruturado durante visitas domiciliares. Os valores de PA dos pacientes foram monitorados durante três semanas consecutivas. A adesão terapêutica foi determinada por meio da aplicação da Escala de Adesão Terapêutica de oito itens de Morisky (MMAS-8). Foram investigados 50 pacientes, com média de idade de 66,4 anos $(\mathrm{DP}=13,5)$, sendo que a maioria dos pacientes era do sexo feminino (74\%). Entre os pacientes estudados, 39 foram 
classificados com hipertensão pseudo-resistente (78\%); destes, 28 utilizavam esquemas anti-hipertensivos considerados inapropriados e 11 eram não aderentes ao tratamento. No presente estudo, $8 \%$ dos hipertensos foram diagnosticados com HAR. Em relação à PA sistólica (PAS) e PA diastólica (PAD), não foi demonstrada a influência de sexo, fumo e atividade física. A prescrição apropriada apresentou relação com o controle da PAS, porém, não apresentou relação com o controle da PAD. O grau de aderência influenciou tanto a PAS como a PAD. Foi observada ainda a influência do subtipo de hipertensão sobre as médias de PAS e PAD.

Palavras-chave: adesão ao medicamento; hipertensão; prescrição inadequada

\section{INTRODUÇÃO}

A hipertensão arterial (HA) está associada direta ou indiretamente a altas taxas de morbidade e mortalidade resultantes das doenças cardiovasculares (DCV) (1). No Brasil, no ano de 2013, as doenças cardiovasculares representaram 29,8\% dos óbitos (2). Além disso, a hipertensão arterial tem indicado prevalências que oscilam de $19,2 \%$ a 44,4\%, porém com baixos índices de controle da doença (3).

O risco de complicações cardiovasculares resultante da HA é crescente a partir de médias de 115 x 75 mmHg. Para cada $20 \mathrm{mmHg}$ acrescidos à pressão arterial sistólica (PAS) ou $10 \mathrm{mmHg}$ para a pressão arterial diastólica (PAD), é observado o dobro do risco, tanto para os eventos de doença arterial coronariana (DAC), quanto para acidente vascular encefálico (AVE). Em razão disto, as classificações do comportamento da pressão arterial têm sido atualmente mais rigorosas, considerando valores inferiores a 140 × $90 \mathrm{mmHg}$ como necessários para grupos específicos de pacientes (4).

Diversas classes de anti-hipertensivos apresentam potenciais benefícios para redução de eventos cardiovasculares (5). No entanto, alguns pacientes não respondem satisfatoriamente às terapias anti-hipertensivas. A Hipertensão Arterial Resistente (HAR) é definida por valores de Pressão Arterial (PA) continuamente acima das metas recomendadas: $130 / 80 \mathrm{mmHg}$ para pacientes com risco elevado de DCV e 140/90mmHg para pacientes de risco baixo e médio, mesmo com o uso de três fármacos anti-hipertensivos nas doses máximas preconizadas e toleradas, sendo um deles preferencialmente um diurético; ou quando em uso de quatro ou mais fármacos anti-hipertensivos, mesmo com a PA sob controle (6). A causa da HAR é também multifatorial, em semelhança à hipertensão arterial, e o tratamento tem por objetivo a identificação e a reversão dos possíveis fatores ambientais que induzem à resistência (7).

Para os pacientes diagnosticados com hipertensão resistente (HAR), a mudança no estilo de vida é funda- mental, estimulando a perda de peso, a diminuição do sal na dieta, a redução ou interrupção da ingestão alcoólica, uma dieta saudável com alta porcentagem de fibras e diminuição da gordura e, principalmente, a atividade física. Esta mudança no estilo de vida é importante para uma ação mais efetiva dos medicamentos anti-hiperten$\operatorname{sivos}(7)$.

Alguns fatores, porém, podem levar a um falso diagnóstico de HAR, caracterizando a hipertensão pseudo-resistente. São eles: não adesão ao tratamento anti-hipertensivo, aferição inadequada da PA, uso de fármacos ou esquemas terapêuticos não apropriados, ou presença do efeito do jaleco branco (6). Na prática clínica, vários pacientes com baixo controle da pressão arterial são classificados inapropriadamente como tendo HAR (8). A avaliação cuidadosa desses pacientes, tendo a vista à identificação da hipertensão pseudorresistente, evita procedimentos desnecessários e dispendiosos, tais como o uso excessivo de anti-hipertensivos $(9,10)$.

Uma melhor compreensão da ocorrência e resultados associados à hipertensão resistente e pseudorresistente é importante para melhorar a gestão desses pacientes, possibilitando um melhor tratamento e podendo evitar possíveis danos ao individuo hipertenso $(11,12)$. Este estudo teve como objetivo identificar a HAR e a hipertensão pseudorresistente em pacientes atendidos em uma Unidade de Saúde da Família.

\section{MÉTODO}

Foi realizado um estudo transversal com cinquenta pacientes hipertensos em uso de medicamentos anti-hipertensivos atendidos na Unidade Básica de Saúde David Nasser, localizada no Bairro de Ipioca, Maceió, em maio de 2013. A seleção dos pacientes se deu por meio de sorteio randômico a partir de uma lista de pacientes elegíveis, fornecida pelos prescritores da unidade de saúde. Foram incluídos pacientes com hipertensão, em uso de medicamentos anti-hipertensivos, com idade 
igual ou acima de 18 anos, cadastrados na unidade de saúde participante da pesquisa. Foram excluídos pacientes que deixaram de residir no endereço cadastrado na UBS durante a realização do estudo.

A coleta de dados foi efetuada por meio de entrevistas com aplicação de questionário estruturado durante visitas domiciliares, além de informações contidas na prescrição médica e no cadastro dos pacientes na UBS. Foram investigadas as seguintes variáveis: sexo, idade, circunferência abdominal, comorbidades, tabagismo, consumo de álcool, prática de atividade física, uso de medicamentos anti-hipertensivos e uso de outros medicamentos.

Os valores de Pressão Arterial Sistólica (PAS) e Diastólica (PAD) foram obtidos ao longo de três semanas pela equipe de pesquisa durante a visita, de acordo com as orientações estabelecidas nas VI Diretrizes Brasileiras para o Tratamento da Hipertensão (2). Foi utilizado um esfigmomanômetro eletrônico digital de braço, calibrado e aprovado pelo INMETRO e pela Sociedade Brasileira de Cardiologia. Foram realizadas duas medidas em cada visita domiciliar, em intervalo mínimo de 10 minutos, em um total de três visitas para cada paciente.

A adesão terapêutica foi determinada por meio da aplicação da Escala de Adesão Terapêutica de oito itens de Morisky (MMAS-8), adaptada para o português e validada em estudo anterior $(13,14)$. Foram considerados aderentes os pacientes cujo escore final foi igual ou maior que seis (15).

A farmacoterapia anti-hipertensiva foi avaliada de acordo com as Diretrizes Brasileiras de Hipertensão (2), sendo considerados (a) fármaco (s) de escolha, (b) doses e posologias, (c) associações e (d) número total de anti-hipertensivos, bem como sua efetividade. Deste modo, demais problemas relacionados a medicamentos, tais como uso de fármacos desnecessários, efeitos adversos (relacionados ou não à dose) e interações medicamentosas, não foram investigados.

A análise dos dados foi realizada utilizando o software SPSS, versão 12. As análises estatísticas envolvidos foram descritiva analisa, o teste de Kolmogorov-Smirnov para verificar a normalidade das variáveis contínuas, qui-quadrado e Kruskal-Wallis para testar a relação entre adesão e outras variáveis independentes, e regressão logística binária. $\mathrm{O}$ nível de significância estabelecido foi $\mathrm{p}=0,05$.

O trabalho foi aprovado pelo Comitê de Ética em Pesquisa da Universidade Federal de Alagoas em
6/11/2009, sob o número 010186/2009-01. Os dados foram coletados somente após a assinatura do termo de consentimento livre e esclarecido por todos os pacientes.

\section{RESULTADOS E DISCUSSÃO}

Foram convidados 50 pacientes para a realização do estudo, com média de idade de 66,4 anos ( $\mathrm{DP}=13,5$ ), idade mínima de 35 e máxima de 85 anos, e todos aceitaram participar, A maioria dos hipertensos estudados era do sexo feminino (74\%). Nenhum paciente possuía escolaridade de nível superior. Sete pacientes (14\%) praticavam atividade física regular (Tabela 1). $\mathrm{O}$ tabagismo e o consumo de álcool foram reportados por $12 \%$ e $4 \%$ dos pacientes, respectivamente. A média da circunferência abdominal foi de 102,2 (DP=14,0), com de mínima de 66 e máxima de $143 \mathrm{~cm}$.

Apenas 30\% dos pacientes tinham alguma comorbidade, sendo as mais comuns a depressão, registrada por 4 pacientes, e a diabetes, encontrada em 2 pacientes. Dos 50 hipertensos analisados, 28 utilizavam esquemas anti-hipertensivos que foram considerados inapropriados (56\%). Destes, 20 pacientes utilizavam associações de anti-hipertensivos inadequadas ou insuficientes e 8 pacientes utilizavam anti-hipertensivos em doses insuficientes.

No presente estudo, foi constatado que $8 \%$ dos hipertensos foram diagnosticados com HAR. No entanto, a maioria foi caracterizada com hipertensão pseudorresistente (78\%).

A média da PAS foi de $149,7 \mathrm{mmHg}$, enquanto a média da PAD foi de $88,1 \mathrm{mmHg}$. Somente 7 pacientes apresentaram todas as medidas de PA sob controle, enquanto que em 27 pacientes, a PA estava fora de controle nos três dias de medida. Em relação ao grau de adesão terapêutica, 13 pacientes apresentaram alto grau de adesão, 25 apresentaram grau médio de adesão e 12 baixa adesão. Pacientes com grau de adesão alto tiveram a PAS controlada $(\mathrm{p}=0,019)$. De acordo com o ponto de corte do escore estabelecido neste estudo, 38 pacientes foram caracterizados como aderentes e tiveram a sua PAD controlada $(\mathrm{p}=0,047)$.

Em relação a PAS e PAD, não foi observada a influência de sexo, fumo e atividade física, nas análises das médias dessas variáveis. Por outro lado, a prescrição apropriada mostrou relação com o controle da PAS, porém, não com o controle da PAD. No entanto, as análises das médias das variáveis do grau de aderência estavam asociadas tanto da PAS como da PAD (Tabela 2). 


\section{Inotarma}

Tabela 1. Relação entre características socioeconômicas, adesão terapêutica, hábitos de vida dos pacientes hipertensos e o controle da PA, de uma Unidade de Saúde da Família em Maceió, 2013.

\begin{tabular}{|c|c|c|c|c|c|c|c|}
\hline \multirow{2}{*}{ Variáveis } & \multirow{2}{*}{$\mathbf{N}$} & \multicolumn{2}{|c|}{ PAS Controlada } & \multirow{2}{*}{$p^{*}$} & \multicolumn{2}{|c|}{ PAD controlada } & \multirow{2}{*}{$p^{*}$} \\
\hline & & $\mathbf{N}$ & $(\%)$ & & $\mathrm{N}$ & $(\%)$ & \\
\hline \multicolumn{8}{|l|}{ Sexo } \\
\hline Fem. & 37 & 14 & $(37,8)$ & & 23 & $(62,2)$ & \\
\hline Masc. & 13 & 3 & $(23,1)$ & 0,334 & 6 & $(46,2)$ & 0,314 \\
\hline \multicolumn{8}{|l|}{ Fumo } \\
\hline Sim & 6 & 3 & $(50)$ & & 3 & $(50)$ & \\
\hline Não & 44 & 14 & $(31,8)$ & 0,379 & 26 & $(59,1)$ & 0,672 \\
\hline \multicolumn{8}{|l|}{ Atividade física } \\
\hline Sim & 7 & 3 & $(42,9)$ & & 4 & $(57,1)$ & \\
\hline Não & 43 & 14 & $(32,6)$ & 0,594 & 25 & $(58,1)$ & 0,960 \\
\hline \multicolumn{8}{|c|}{ Prescrição apropriada } \\
\hline Sim & 22 & 10 & $(45,5)$ & & 13 & $(59,1)$ & \\
\hline Não & 28 & 7 & (25) & 0,130 & 16 & $(57,1)$ & 0,890 \\
\hline \multicolumn{8}{|l|}{ Adesão } \\
\hline Sim & 38 & 13 & $(34,2)$ & & 25 & $(65,8)$ & \\
\hline Não & 12 & 4 & $(33,3)$ & 0,955 & 4 & $(33,3)$ & 0,047 \\
\hline \multicolumn{8}{|l|}{ Grau de adesão } \\
\hline Score $=8$ & 12 & 8 & $(66,7)$ & & 10 & $(83,3)$ & \\
\hline Score $\geq 6$ & 25 & 5 & (20) & & 14 & $(56)$ & \\
\hline Sore $<6$ & 13 & 5 & $(30,8)$ & 0,019 & 5 & $(38,5)$ & 0,073 \\
\hline
\end{tabular}

Tabela 2. Associação entre o controle da PAS e PAD média das variáveis nos pacientes hipertensos e o controle da PA, de uma Unidade de Saúde da Família em Maceió, 2013.

\begin{tabular}{|c|c|c|c|c|}
\hline Variáveis & Média da PAS & p* & Média da PAD & p* \\
\hline \multicolumn{5}{|l|}{ Sexo } \\
\hline Fem. & 148,58 & & 87,43 & \\
\hline Masc. & 152,79 & 0,456 & 90,05 & 0,483 \\
\hline \multicolumn{5}{|l|}{ Fumo } \\
\hline Sim & 139,78 & & 84,39 & \\
\hline Não & 151,03 & 1,36 & 88,62 & 0,400 \\
\hline \multicolumn{5}{|l|}{ Atividade física } \\
\hline Sim & 145,86 & 0,534 & 86,19 & \\
\hline Não & 150,30 & & 88,43 & 0,636 \\
\hline \multicolumn{5}{|l|}{ Prescrição apropriada } \\
\hline Sim & 143,71 & & 86,42 & \\
\hline Não & 154,37 & 0,029 & 89,44 & 0,360 \\
\hline \multicolumn{5}{|l|}{ Adesão } \\
\hline Sim & 149,41 & & 86,73 & \\
\hline Não & 150,53 & 0,848 & 92,50 & 0,129 \\
\hline \multicolumn{5}{|l|}{ Grau de adesão } \\
\hline Score $=8$ & 136,17 & & 79,97 & \\
\hline Score $\geq 6$ & 156,11 & & 89,84 & \\
\hline Score $<6$ & 149,79 & 0,003 & 92,31 & 0,012 \\
\hline
\end{tabular}


As variáveis sexo, fumo, atividade física e prescrição apropriada não apresentaram relação com o controle da PAS e da PAD. Contudo, foi observada relação entre adesão e controle da PAD em consequência da mudança de ponto de corte na MMAS-8, que considera o paciente aderente ao tratamento anti-hipertensivo quando obtém o ponto de corte no score igual ou maior que 6 . $\mathrm{O}$ grau de adesão tem relação com a PAS $(\mathrm{p}=0,0019)$ e fica próximo da significância em comparação com a $\operatorname{PAD}(\mathrm{p}=0,073)$.

A Tabela 3 mostra que houve influência no subtipo de hipertensão em relação às médias de PAS e ${ }^{\mathrm{a}} \mathrm{PAD}$. Foi observado que pacientes classificados com hipertensão pseudorresistente do tipo mista apresentaeram médias de PAS e PAD piores que pacientes também pseudorresistente que foram classificados como não aderentes ou com prescrição inadequada $(\mathrm{P}=0,001$ e 0,014 respectivamente). No presente estudo, nenhum paciente com a PA controlada seguia um tratamento medicamentoso inapropriado (Tabela 3).

Novas orientações têm apontado a utilização da monitoração ambulatorial da pressão arterial (MAPA) e da monitoração residencial da pressão arterial (MRPA) como ferramentas importantes na determinação da PA (2). No entanto, estes métodos demandam recursos financeiros ou tempo, reduzindo sua aplicabilidade prática. A abordagem proposta no presente estudo - três medidas isoladas, cada uma obtida por uma média de duas medidas por visita, ao longo de 3 semanas - permitiu a identificação de pacientes com baixo controle sustentado da PA, associada a uma prevalência de HAR semelhante àquela encontrada em estudo anterior realizado no Brasil (16), no qual o MAPA foi utilizado.
Alguns achados podem ser inicialmente observados no perfil socioeconômico e nos hábitos de vida da população estudada. Vários estudos realizados com pacientes hipertensos no Brasil indicam maior prevalência de idosos (17-19), do sexo feminino $(17,20)$ e baixa escolaridade $(18,19,21,22)$. Em um estudo transversal (23), foram observadas características socioeconômicas semelhantes ao presente estudo.

\section{CONCLUSÃO}

No presente estudo, menos de $20 \%$ dos pacientes apresentou valores de pressão arterial continuamente dentro das metas terapêuticas. A prevalência da hipertensão resistente foi semelhante à prevalência identificada em estudos anteriores, tanto no Brasil quanto em outros países. A maioria dos pacientes apresentou hipertensão pseudorresistente, sendo o subtipo mais comum a hipertensão pseudorresistente causada por baixa adesão terapêutica. No entanto, o uso de farmacoterapia anti-hipertensiva inapropriada se relacionou aos piores desfechos em toda amostra: os pacientes com hipertensão pseudorresistente causada por prescrição inapropriada apresentaram valores médios de pressão arterial sistólica e diastólica superiores aos valores médios dos pacientes com hipertensão resistente. Deste modo, a identificação de pacientes com hipertensão pseudorresistente, além de otimizar o diagnóstico da hipertensão resistente, evitando tratamentos desnecessários, pode levar a uma maior efetividade no controle da pressão arterial desde que priorizada a otimização da farmacoterapia.

Tabela 3. Relação entre os subtipos de hipertensão e as médias de PAS e PAD em pacientes hipertensos e o controle da PA, de uma Unidade de Saúde da Família em Maceió, 2013.

\begin{tabular}{|c|c|c|c|c|}
\hline Variáveis & Média da PAS & $p^{*}$ & Média da PAD & $\mathrm{p}^{*}$ \\
\hline \multicolumn{5}{|l|}{ Subtipo de hipertensão } \\
\hline PA Ctrl* & 123,8 & & 73,7 & \\
\hline PA Ctrl $(\mathrm{NADH})^{* *}$ & 133,7 & & 82,0 & \\
\hline $\mathrm{PA} C \operatorname{trl}(\mathrm{PI})^{\star * *}$ & - & & - & \\
\hline PA Ctrl (PI) e (NADH)+ & - & & - & \\
\hline $\mathrm{HAR}^{++}$ & 153,2 & & 87,3 & \\
\hline HPR $(\mathrm{NADH})^{+++}$ & 152,1 & & 93,4 & \\
\hline $\operatorname{HPR}(\mathrm{PI})^{\triangle}$ & 153,5 & & 89,1 & \\
\hline HPR (mista) ${ }^{\Delta \Delta}$ & 178,3 & 0,001 & 97,3 & 0,014 \\
\hline
\end{tabular}

"Pressão arterial controlada. ${ }^{* *}$ Pressão arterial controlada com não-adesão ao tratamento. ${ }^{* * *}$ Pressão arterial controlada com prescrição inapropriada.

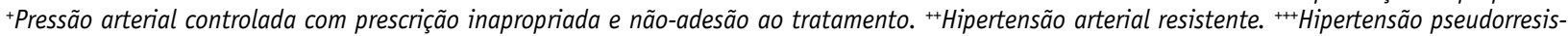
tente causada por não-adesão ao tratamento. ${ }^{\Delta}$ Hipertensão pseudorresistente ocasionada por prescrição inadequada. ${ }^{\Delta \Delta} \mathrm{Hipertensão} \mathrm{pseudorresistente}$ causada tanto por prescrição inapropriada quanto por não-adesão ao tratamento. 


\section{REFERÊNCIAS}

1. Nobre F, Ribeiro AB, Mion D. Controle da pressão arterial em pacientes sob tratamento anti-hipertensivo no Brasil. Arq Bras Cardiol. 2010;94 (5):663-670. DOI: 10.1590/S0066-782X2010005000035

2. SBC. Sociedade Brasileira de Cardiologia. VI Diretrizes Brasileiras de Hipertensão. Arq Bras Cardiol. 2010;95(1 Suppl 1): 1-51. DOI:10.1590/S0066782X2010001700001.

3. Passos VMA, Assis TD, Barreto SM. Hipertensão arterial no Brasil: estimativa de prevalência a partir de estudos de base populacional Epidemiologia e Serviços de Saúde. Epidemiol Serv Saúde. 2006;15(1):35-45.

4. Lewington S, Clarke R, Chizibash N, Peto R, Collins R. Prospective Study Collaboration. Age specific relevance of usual blood pressure to vascular mortality: a meta-analysis of individual data for one million adults in 61 prospective studies. Lancet. 2002;360(14):1903-1913. DOI: 10.1016/S0140-6736(02)11911-8

5. Martins LC, Martins LMB, Júnior SUHM. Tratamento medicamentoso do paciente com hipertensão de difícil controle. Rev Bras Hipertens. 2008; 15(1): 28-33

6. SBC. Sociedade Brasileira de Cardiologia. I Posicionamento Brasileiro sobre Hipertensão Arterial Resistente. Arq Bras Cardiol. 2012; 99 (1): 576-585. DOI: 10.1590/ S0066-782X2012001000002

7. Póvoa R, Scala LCN, Moreno HF. Estratégias medicamentosas na hipertensão arterial resistente. Rev Bras Hipertens. 2009; 16 (Suppl 1): S10-S12.

8. Volpe M, Tocci G. Challenging hypertension: how to diagnose and treat resistant hypertension in daily clinical practice. Rev Cardiovasc. 2010; 8(6): 811-820. DOI: 10.1586/erc. 10.47

9. Oliveira-Filho, AD, Costa FA, Neves SJ, Lyra Junior DP, Morisky DE. Pseudoresistant hypertension due to poor medication adherence. Int J Cardiol. 2014; 172(2):309310. DOI: 10.1016/j.ijcard.2013.12.181.

10. Vieira AJ. Resistant Hypertension. J Am Board Fam Med. 2012; 25(4):487-495. DOI: 10.3122/jabfm.2012.04.110275.

11. Daugherty SL, Powers D, Magid DJ, Tavel HM, Masoudi FA, Margolis KL, O’Connor PJ, Selby JV, Ho PM. Incidence and Prognosis of Resistante Hypertension in Hipertensive Patientes. Circulation. 2012;125:1635-1642. DOI: 10.1161/CIRCULATIONAHA.111.068064

12. Judd E, Calhoun D. Apparent and true resistant hypertension: definition, prevalence and outcomes. J Hum Hypertens. 2014; 28(8):463-468. DOI:10.1038/jhh.2013.140.

13. Oliveira-Filho AD, Barreto-Filho JAB, Neves SJF, Lyra Junior DPL. Association between the 8-item Morisky Medication Adherence Scale (MMAS-8) and blood pressure control. Arq Bras Cardiol. 2012; 99 (1): 649-658.

14. Oliveira-Filho AD, Morisky DE, Neves SJ, Costa FA, Lyra Junior DP. The 8-item Morisky Medication Adherence Scale: Validation of a Brazilian-Portuguese version in hypertensive adults. Res Social Adm Pharm. 2013; 10 (3): 554-561. DOI: 10.1016/j.sapharm.2013.10.006.
15. Morisky DE, Ang A, Krousel-Wood M, Ward HJ. Predictive validity of a medication adherence measure in an outpatient setting. J Clin Hypertens. 2008; 10(5):348-354. DOI: $10.1111 / \mathrm{j} .1751-7176.2008 .07572 . x$

16. Massierer D, Oliveira ACT, Steinhorst AM, Gus M, Ascoli AM, Gonçalves SC, Moreira LB, Correa-Junior V, Nunes G, Fuchs SC, Fuchs FD. Prevalência de hipertensão resistente em adultos não idosos: estudo prospectivo em contexto ambulatorial. Arq Bras Cardiol. 2012; 99 (1):630-635. DOI: 10.1590/S0066-782X2012005000051

17. Firmo JOA, Uchôa E, Lima-Costa MF. Projeto Bambuí: fatores associados ao conhecimento da condição de hipertenso entre idosos. Cad Saude Publica. 2004;20(2):512521.

18. Nascente FMN, Jardim PCBV, Peixoto MDCG, Monego ET, Moreira HG, Vitorino PVDO. Hipertensão arterial e sua correlação com alguns fatores de risco em cidade brasileira de pequeno porte. Arq. Bras. Cardiol. 2010;95(4): 502-509. DOI: 10.1590/S0066-782X2010005000113

19. Feijão AMM, Gadelha FV, Bezerra AA, Oliveira AM, Silva MSS, Lima JWO. Prevalência de excesso de peso e hipertensão arterial, em população urbana de baixa renda. Arq. Bras. Cardiol. 2005; 84(1): 29-33. DOI: 10.1590/ S0066-782X2005000100007

20. Ormigli VLA, Jacobina RR, Noblat ACB, Sobrinho CLN, Noblat LACB, Souza LF, Chaves CR, Reis MA, Nascimento LD, Rocha AD, Ramos CMO. Hipertensão arterial em adultos de um bairro de Salvador, Bahia. Rev Baiana Saúde Pública.1999;23(1):7-20.

21. BRASIL. Ministério da Saúde. Instituto Nacional do Câncer. Inquérito domiciliar sobre comportamento de risco e morbidade referida de doenças e agravos não transmissíveis. Rio de Janeiro: INCA; 2005. [acesso 2005 mar. 14, informações em publicação sobre o inquérito]. Disponível em: http://www.inca.gov.br

22. Souza ARA, Costa A, Nakamura D, Mocheti LN, Stevanato FPR, Ovando LA. Um estudo sobre hipertensão arterial sistêmica na cidade de Campo Grande, MS. Arq. Bras. Cardiol. 2007; 88(4): 441-446. DOI:10.1590/ S0066-782X2007000400013.

23. Selem SSC, Castro MA, César CLG, Marchioni DML, Fisberg RM. Validade da hipertensão autorreferida associa-se inversamente com escolaridade em brasileiros. Arq. Bras. Cardiol. 2013; 100(1):52-59. DOI: 10.1590/ S0066-782X2012005000119.

24. Persell SD. Prevalence of resistant hypertension in the United States, 2003-2008. J Am Heart Assoc. 2011; 57(6):1076-1080. DOI:10.1161/HYPERTENSIONAHA.111.170308.

25. Oigman W, Fritsch MNT, Sanjuliani AF. Curva-J: Conceito, considerações fisiopatológicas e efeito da terapia anti-hipertensiva. Rev Bras Hipertens; 2010; 17(3):156159.

26. Pimenta, E., \& Calhoun, D. A. (2012). Resistant Hypertension: Incidence, Prevalence and Prognosis. Circulation, 125(13), 1594-1596. DOI: 10.1161/CIRCULATIONAHA.112.097345. 